\title{
Outcomes After Proton Therapy Reirradiation for Malignant Glioma: Prospective Analysis of Over 100 Patients from the Proton Collaborative Group
}

Robert H Press ( $\square$ rpress@nyproton.com )

New York Proton Center https://orcid.org/0000-0002-3297-3945

Shaakir Hasan

New York Proton Center

$\mathrm{J}$ Isabelle Choi

New York Proton Center

Arpit M Chhabra

New York Proton Center

Lia M Halasz

University of Washington

Carlos E Vargas

Mayo Clinic Arizona

Henry K Tsai

ProCure Proton Therapy Center

Chiachien J Wang

Willis-Knighton Medical Center

John H Chang

The Oklahoma Proton Center

Vinai Gondi

Northwestern Medical Center

Mark V Mishra

University of Maryland School of Medicine

Charles B Simone II

New York Proton Center

\section{Research Article}

Keywords: malignant glioma, reirradiation, proton therapy, glioblastoma, toxicities

Posted Date: May 11th, 2021

DOI: https://doi.org/10.21203/rs.3.rs-499865/v1 
License: (c) (i) This work is licensed under a Creative Commons Attribution 4.0 International License. Read Full License 


\section{Abstract}

Purpose: The optimal salvage treatment for malignant glioma after prior radiotherapy (RT) is not well established. Proton therapy (PT) is an attractive treatment modality for reirradiation (ReRT) of recurrent disease; however, data are limited.

Methods: The prospective, multi-institutional Proton Collaborative Group (PCG) registry was queried for patients with malignant glioma who previously received RT and underwent PT-ReRT between 7/2011$10 / 2018$. Overall survival (OS) and progression-free survival (PFS) were assessed using the Kaplan-Meier method and Cox proportional hazards regression analysis.

Results: 109 consecutive patients were identified. Median follow-up was 11.2 months and median time interval (TI) from prior RT (median 59.3 Gy) to PT-ReRT (median $49.6 \mathrm{~Gy}$ ) was 45.7 months. Primary histology was glioblastoma $(n=62)$, oligodendroglioma $(n=26)$, and astrocytoma $(n=20)$. Median PFS and OS was 6.1 and 11.1 months, respectively. On UVA, improved PFS was associated with oligodendroglioma (HR 0.39) and astrocytoma (HR 0.55) histologies, WHO Grade III (HR 0.36), TI (HR 0.989), surgical resection (HR 0.39), and PT-ReRT dose $\geq 50$ Gy (HR 0.59) (all $p<0.03$ ), while improved OS was associated with oligodendroglioma histology (HR 0.35), WHO grade III (HR 0.33), ECOG 0 (HR 0.47), TI (HR 0.985), and PT-ReRT dose $\geq 50$ Gy (HR 0.58) (all p<0.04). Late Grade 3 toxicities occurred in $5.6 \%$ of patients with no attributable acute or late Grade 4-5 events.

Conclusion: In the largest series of glioma PT-ReRT reported to date, retreatment appears well tolerated with acceptable outcomes and favorable toxicities compared to photon historical controls. Dose escalation achievable with PT may improve outcomes for well-selected patients.

\section{Introduction}

Malignant glioma represents $25 \%$ of all primary brain tumors.[1] Unfortunately, despite standard therapies, malignant glioma inevitably recurs, most commonly in-field and/or marginal to the original resection bed and radiotherapy (RT) treatment fields.[2, 3] In the recurrent setting, outcomes and optimal salvage therapies are not well established. Treatment options include maximally safe re-resection, systemic therapies, tumor treating fields, and reirradiation.[4] A second course of RT can be considered with the goal of providing durable control of the recurrent tumor and preventing the associated morbidity of local tumor progression.

Reirradiation of recurrent glioma can be challenging due to the previous receipt of high-dose RT in close proximity to critical organs at risk (OAR), such as the brainstem and optic structures. Excess cumulative dose to OARs and normal non-target brain tissue may increase the risk of significant neurologic sequela and potential detriment to patient quality of life (QOL).[5-9] As such, administration of definitive reirradiation doses are not typically feasible due to risks of high-grade toxicities. 
Proton therapy (PT) is an auspicious treatment modality for this clinical scenario.[10] The physical properties of PT compared to photon therapy offer greater capability to cover the recurrent disease with meaningful radiation dose while minimizing excess exit-dose to OARs and normal brain tissue. PT may, therefore, facilitate safer and more effective treatment in the recurrent setting and, in a select minority of cases, offer patients a new chance of durable disease control.[11] In this context, the goal of this study was to analyze patients with recurrent glioma enrolled on the prospective multi-institutional Proton Collaborative Group (PCG) registry for treatment patterns, patient outcomes, and treatment-related toxicities.

\section{Methods}

The PCG is a research consortium of 17 proton centers in the United States. REG001-09 (NCT01255748) is a prospective registry study for which each institution obtained individual IRB approval. The study opened for accrual in 2010 and is currently enrolling patients. The registry was queried for all consecutive patients with recurrent glioma treated with PT-reirradiation from 2011-2018. Patient and tumor characteristics, prior and current radiation details, clinical outcomes, and toxicities were extracted and reviewed retrospectively.

Overall survival (OS) and progression-free survival (PFS) were defined as time from initiation of PTreirradiation to the event of interest and censored at the time of last follow-up or death. These results were recorded individually by each institution and reviewed centrally by PCG staff. Descriptive statistics were used to report patient, tumor, and treatment characteristics. Outcomes were assessed using the Kaplan-Meier method and both univariable and multivariable Cox proportional hazards regression analysis, denoted as hazard ratios (HR) with 95\% confidence intervals (Cl). Demographic and treatment details included in the univariable analysis (UVA) were age, sex, race, tumor location, histology, grade, IDH mutation status, 1p19q co-deletion, MGMT methylation status, ECOG performance status, tumor size, surgical resection, extent of resection, systemic therapy use, RT dose and fractionation, and time interval from prior RT to PT-reirradiation (as a continuous variable). Each variable with a p-value $<0.1$ was included in the multivariable analysis (MVA). Toxicities were scored according to CTCAE v4.0. Univariable binomial regression analysis was performed to identify clinical correlates of toxicity. To account for differences in dose fractionation, radiation dose was summed via equivalent dose in 2 Gy fractions (EQD2) using a representative $\alpha / \beta$ ratio of 10 . The statistical program used was MedCalc version 19.1 (Ostend, Belgium). All statistical analyses were performed using a $p$-value significance level of $<0.05$.

\section{Results}

Patient, tumor, and treatment characteristics are listed in detail in Table 1. In the database, 109 patients with recurrent glioma treated with PT across 7 treatment centers were identified. Of those, $50 \%(n=55)$ were male, $80 \%(n=87)$ were white, and median age was 49 years (range 19-85). Most patients had an ECOG performance status of $0(n=48)$ or $1(n=37)$. Primary tumor histology was glioblastoma $(n=63$, including 1 gliosarcoma), oligodendroglioma $(n=26)$, and astrocytoma $(n=20)$. Of the non-glioblastoma 
patients, 28 had WHO grade III tumors, including 16 with anaplastic oligodendroglioma and 12 with anaplastic astrocytoma. Molecular data were not commonly available for non-glioblastoma patients. Of those with available data, IDH1 mutation and MGMT methylation was present in $20 \%(n=8$ of 41$)$ and $52 \%$ of patients $(n=22$ of 42$)$, respectively. 
Table 1

Patient and treatment characteristics

\begin{tabular}{|c|c|c|}
\hline Parameter & Level & $N(\%)=109$ \\
\hline Age (years) & Median (Range) & $49(19-85)$ \\
\hline \multirow[t]{2}{*}{ Sex } & Male & $55(50 \%)$ \\
\hline & Female & $54(50 \%)$ \\
\hline \multirow[t]{4}{*}{ ECOG } & 0 & $48(44 \%)$ \\
\hline & 1 & $37(34 \%)$ \\
\hline & 2 & $18(17 \%)$ \\
\hline & 3 & $6(5 \%)$ \\
\hline \multirow[t]{3}{*}{ Histology at Diagnosis } & Glioblastoma & $63(58 \%)$ \\
\hline & Astrocytoma & $20(18 \%)$ \\
\hline & Oligodendroglioma & $26(24 \%)$ \\
\hline \multirow[t]{3}{*}{ Tumor Grade } & 4 & $63(58 \%)$ \\
\hline & 3 & $28(26 \%)$ \\
\hline & 2 & $18(16 \%)$ \\
\hline \multirow[t]{2}{*}{ Prior Surgical Resection } & Yes & $94(86 \%)$ \\
\hline & No & $15(14 \%)$ \\
\hline \multirow[t]{2}{*}{ Prior Chemotherapy } & Yes & 97 (89\%) \\
\hline & No & $12(11 \%)$ \\
\hline \multirow[t]{2}{*}{ Surgical resection within 3 months of PT } & Yes & $33(40 \%)$ \\
\hline & No & $76(70 \%)$ \\
\hline \multirow[t]{3}{*}{ Number of prior RT courses } & 1 & $102(94 \%)$ \\
\hline & 2 & $6(5 \%)$ \\
\hline & 3 & $1(1 \%)$ \\
\hline Prior RT Dose (Gy) & Median (Range) & $59.3(23.4-120.9)$ \\
\hline Time interval from prior RT to PT (months) & Median (Range) & $40.7(2.9-441.0)$ \\
\hline PT Reirradiation Dose (CGE in Gy) & Median (Range) & $49.6(24.8-60.1)$ \\
\hline Fraction Size (CGE in Gy) & Median (Range) & $2.2(1.1-4.0)$ \\
\hline PT Modality & Pencil-beam scanning & $56(52 \%)$ \\
\hline
\end{tabular}




\begin{tabular}{|lll|}
\hline Parameter & Level & $\mathbf{N}(\%)=109$ \\
\hline Cumulative Dose (CGE in Gy) & Passive scattering & $53(48 \%)$ \\
\hline
\end{tabular}

Overall, $86 \%$ and $89 \%$ of patients had surgery and chemotherapy at initial diagnosis, respectively. Most patients received temozolomide ( $n=73$ of 97). Most patients had a single prior course of RT $(n=102)$, but 6 patients had 2 prior courses and 1 patient had 3 prior courses. The median cumulative dose of all prior treatments was $59.3 \mathrm{~Gy}$ (range 23.4-120.9). Most prior treatment courses were conventional and/or mildly hypofractionated dose fractionation regimens; however, $5 \%$ of patients received stereotactic radiosurgery at least once prior to $\mathrm{PT}$.

Of the patients with one prior course of RT, the median time from initial diagnosis to recurrence was 45.7 months (range 3.2-386.7 months). For the whole cohort, $40 \%$ of patients had surgery within 3 months of PT. The median time from the most recent prior RT course to initiation of PT-reirradiation for the entire cohort and the glioblastoma cohort was 40.7 months (range 2.9-441.0) and 14.7 months (range 3.989.5), respectively. Pencil beam scanning PT $(n=56)$ and uniform scanning PT $(n=53)$ were utilized. The median dose of PT-reirradiation was 49.6 Gy (CGE) (range 24.9-60.1) with a median dose/fraction size of 2.2 CGE/fraction (range 1.1-4.0). The median cumulative dose of all RT courses was $106.5 \mathrm{~Gy}$ (CGE) (range 78.8-167.8). Sixty-eight percent of patients had concurrent chemotherapy with PT-reirradiation. Of those who received concurrent chemotherapy, the most common agents included temozolomide $(n=36)$, bevacizumab $(n=12)$, and temozolomide/bevacizumab combination $(n=21)$.

Progression-free survival and overall survival

Outcomes analyses were performed for patients with only one prior course of RT $(n=102)$. The median follow-up time for surviving patients was 11.2 months. The median PFS was 6.1 months $(95 \% \mathrm{Cl} 5.1-$ 8.5), and the PFS at 3,6 , and 12 months were $74.5 \%, 53.8 \%$ and $23.6 \%$, respectively. Median PFS was longer for oligodendroglioma ( 10.7 months, $95 \% \mathrm{Cl} 5.8-15.8)$ and astrocytoma histologies (10.5 months, 95\% Cl 2.5-21.6) than for glioblastoma (5.2 months, 95\% Cl 3.5-20.2) (Fig. 1a). On UVA, improved PFS was associated with oligodendroglioma ( $\mathrm{HR} 0.39,95 \% \mathrm{Cl} 0.22-0.67, \mathrm{p}=0.0033)$ and astrocytoma histologies (HR $0.55,95 \% \mathrm{Cl} 0.30-0.99, \mathrm{p}=0.0042)$ compared to glioblastoma, WHO grade III compared to WHO grade IV (HR 0.36, 95\% $\mathrm{Cl} 0.21-0.59, \mathrm{p}=0.002)$, longer time to recurrence (HR $0.989,95 \% \mathrm{Cl}$ $0.984-0.995, p=0.0005)$, surgery within 3 months ( $\mathrm{HR} 0.39,95 \% \mathrm{Cl} 0.21-0.73, p=0.0031)$, and reirradiation dose $\geq 50$ Gy (CGE) (HR 0.59, 95\% Cl 0.37-0.95, $p=0.0284$ ) (Table 2a). Median PFS for patients receiving $\geq 50$ Gy (CGE) was 9.1 months compared to 5.1 months for those receiving < 50 Gy (CGE) (Fig. 2A). On multivariable analysis (MVA), time to recurrence (HR 0.991, 95\% Cl 0.984-0.997, $\mathrm{p}=$ 0.0041 ) was maintained within the model. 
Table 2

Univariable and Multivariable Analysis for (a) Progression-Free Survival and (b) Overall Survival

\section{Univariable Analysis}

Variable

Level

Hazard Ratio

$(95 \% \mathrm{Cl})$
Multivariable Analysis

P. value
Hazard Ratio

$(95 \% \mathrm{Cl})$
P.

value

(a) Progression-Free Survival

$\begin{array}{llll}\text { Histology } & \text { Oligodendroglioma } & 0.39(0.22- & 0.0033 \\ & & 0.67) & \\ & \text { Astrocytoma } & \begin{array}{l}0.55(0.30- \\ \end{array} & 0.99) \\ & \text { Glioblastoma } & \text { REF } & \\ & & & \end{array}$

Tumor Grade

WHO Grade 3

$0.36(0.21-$

$0.59)$

0.002

WHO Grade 4

REF

Time to recurrence

(months)

Continuous

variable

$0.989(0.984-\quad 0.0005$

0.995)

0.986 (0.977-

0.995)

0.0015

Surgery within 3

months

Yes

$0.39(0.21-$

$0.73)$

0.0031

No

REF

PT Reirradiation

Dose

$$
\geq 50 \text { Gy }
$$

0.59 (0.37-

0.028

$<50$ Gy

REF

(b) Overall Survival

\begin{tabular}{llllll} 
Histology & Oligodendroglioma & $\begin{array}{l}0.35(0.19- \\
0.62)\end{array}$ & 0.0047 & & \\
& Glioblastoma & REF & & & \\
ECOG & 0 & $\begin{array}{l}0.47(0.27- \\
0.81)\end{array}$ & 0.007 & $0.42(0.19-$ & 0.04 \\
& $\geq 1$ & REF & & & $0.96)$ \\
& & $0.985(0.977-$ & 0.0002 & $0.986(0.978-$ & 0.007 \\
$\begin{array}{l}\text { Time to recurrence } \\
\text { (months) }\end{array}$ & $\begin{array}{l}\text { Continuous } \\
\text { variable }\end{array}$ & $0.993)$ & & $0.994)$ & \\
$\begin{array}{l}\text { PT Reirradiation } \\
\text { Dose }\end{array}$ & $\geq 50$ Gy & $0.58(0.35-$ & 0.039 & & \\
& & $0.97)$ & & & \\
& $<50$ Gy & REF & & & \\
\hline
\end{tabular}


The median OS after PT-reirradiation was 11.1 months (95\% Cl 9.1-12.5) and OS at 6, 12, and 24 months were $75.3 \%, 45.8 \%$, and $16.3 \%$, respectively. Median OS was longer for patients with oligodendroglioma (22.9 months, 95\% Cl 9.5-29.4) and astrocytoma histologies (16.3 months, 95\% Cl 4.3-25.6) compared to glioblastoma (9.5 months, $95 \% \mathrm{Cl} 6.7-38.1$ ) (Fig. 1b). The median OS from time of original diagnosis for the entire cohort, oligodendroglioma, astrocytoma, and glioblastoma histologies were 57.7 months (95\% Cl 38.8-73.8), 169.0 months (95\% Cl 102.6-169.0), 131.4 months ( $95 \% \mathrm{Cl} 43.7-170.7)$, and 33.0 months (95\% Cl 23.2-49.9), respectively. On UVA, improved OS was associated with oligodendroglioma histology compared to glioblastoma (HR $0.35,95 \% \mathrm{Cl} 0.19-0.62, \mathrm{p}=0.0047$ ), WHO grade III compared to WHO grade IV (HR 0.33, 95\% Cl 0.19-0.56, $\mathrm{p}=0.0029)$, ECOG performance status $0(\mathrm{HR} 0.47,95 \% \mathrm{Cl}$ $0.27-0.81, p=0.007)$, longer time to recurrence ( $\mathrm{HR} 0.985,95 \% \mathrm{Cl} 0.977-0.993, \mathrm{p}=0.0002)$, and reirradiation dose $\geq 50$ Gy (CGE) (HR 0.58, 95\% Cl 0.35-0.97, $\mathrm{P}=0.039$ ) (Table 2b). Median OS for patients receiving $\geq 50$ Gy (CGE) was 14.4 months compared to 9.5 months for those receiving < 50 Gy (CGE) (Fig. 2b). Surgery within 3 months was not found to be significant for OS (HR 0.69, 95\% Cl 0.37$1.3, p=0.25)$. On MVA, time to recurrence (HR 0.986, 95\% $\mathrm{Cl} 0.978-0.994, p=0.0007)$ and ECOG 0 (HR $0.42,95 \% \mathrm{Cl} 0.19-0.96, \mathrm{p}=0.04$ ) were maintained within the model.

\section{Toxicity}

At baseline just prior to reirradiation, 12 patients had documented grade 3 toxicities (11\%) from prior therapies. Acute grade $2+$ and grade $3+$ toxicities possibly, probably, or definitely-related to PTreirradiation were reported in $53 \%$ and $10.5 \%$ of patients, respectively. Acute grade 3 toxicities included ataxia $(n=4)$, fatigue $(n=1)$, headache $(n=3)$, weakness $(n=6)$, neuropathy $(n=1)$, and seizure $(n=1)$. There was one acute grade 4 event of thrombocytopenia in a patient receiving concurrent temozolomide. On UVA, the only variable associated with acute grade 3 toxicity was ECOG 2 (HR 2.86, 95\% Cl 0.58-5.14, $p=0.012)$.

Of the 72 patients with $>3$ months follow-up, late grade $2+$ and 3 toxicities occurred in $18.1 \%$ and $5.6 \%$ of patients, respectively. Late grade 3 toxicities included muscle weakness $(n=2)$, memory impairment $(n=$ $1)$, ataxia $(n=1)$, dysarthria $(n=1)$, peripheral neuropathy $(n=1)$, and seizure $(n=1)$. No late grade $4-5$ toxicities were observed. Of the 4 patients with late grade 3 toxicities, the median dose of PT-reirradiation and cumulative dose of all prior RT courses was $43.1 \mathrm{~Gy}$ (CGE) and 102.1 Gy (CGE), respectively.

\section{Discussion}

The current study describes the largest series of patients with recurrent malignant glioma treated to date with PT-reirradiation. This multi-institutional prospectively-collected dataset demonstrates comparable efficacy to photon reirradiation series while achieving a favorable toxicity profile. Our study corroborates several known prognostic factors of recurrent malignant glioma, including tumor histology/grade, performance status, and time to recurrence. In addition, outcomes appear superior when utilizing reirradiation dose of $>50 \mathrm{~Gy}$ (CGE). This finding suggests a role for dose escalation in well selected 
patients with favorable clinical features and good performance status, a treatment paradigm which may be particularly suited for PT.

Recurrent glioma is a challenging clinical scenario. Most patients have already received high-dose chemoradiotherapy, commonly to 50.4-60 Gy, and tumors are often in close proximity to critical neurologic OARs that already received radiation doses at or close to tolerance. In addition, as noted in our study, patients commonly already suffer from neurological sequalae related to prior therapies as well as ongoing tumor-related deficits. Subsequent treatment decisions can, therefore, be exceptionally difficult to make.

There is currently a robust collection of studies examining photon reirradiation for recurrent glioma, particularly for recurrent WHO Grade IV glioblastoma. $[12,13]$ Numerous retrospective series most commonly utilize modest dose regimens ranging from 30-37.5 Gy/10-15 fractions. These series demonstrate relatively consistent rates of survival (e.g. median OS of 7.9-13 months for glioblastoma) with acceptable rates of high-grade toxicity.[14-20]

Combs et al. included 172 patients with malignant glioma treated with a median dose of $36 \mathrm{~Gy} / 13$ fractions and reported median OS of 8 months, 16 months, and 22 months for WHO grade IV, III, and II tumors, respectively.[14] In addition to histology, OS was also associated with time to recurrence, consistent with our analysis. Fogh et al. reported 147 patients with recurrent glioblastoma undergoing reirradiation to $35 \mathrm{~Gy} / 10$ fractions. Median OS for this cohort was 11 months, and only one patient (0.6\%) developed a late grade 3 event.[15] Survival outcomes in these studies were comparable to our results, which demonstrated a median OS of 9.5 months, 16.3 months, and 22.9 months for patients with glioblastoma, astrocytoma, and oligodendroglioma, respectively. RTOG 1205, a randomized phase-II trial comparing bevacizumab alone or bevacizumab with hypofractionated reirradiation to $35 \mathrm{~Gy} / 10$ fractions for recurrent glioblastoma, was recently presented in abstract form, demonstrating a clear PFS benefit (7.1 months vs. 3.8 months) but no difference in OS (10.1 months vs. 9.7 months) with combinedmodality therapy. Despite this, toxicity was quite low after reirradiation, with acute and late grade $3+$ events for the whole cohort of $5 \%$ and $0 \%$, respectively.[21] Another randomized trial was recently conducted by Henry Ford Hospital comparing bevacizumab alone to bevacizumab with fractionated radiosurgery to $32 \mathrm{~Gy} / 4$ fractions with similar clinical and toxicity outcomes.[22]

These studies appear to confirm the safety profile and potential to delay disease progression for modestly-dosed hypofractionated reirradiation regimens. This strategy may be particularly useful for high-grade recurrences. Despite a tumor control benefit, survival outcomes remain disappointing. With low reported rates of treatment-related toxicity, dose intensification may be a reasonable strategy to enhance reirradiation efficacy within the therapeutic window.

To date, reports using definitive or near-definitive reirradiation dose regimens are much more limited, likely due to concerns for treatment-related toxicities. Historical studies found reirradiation doses $>40$ Gy using conventional 3D-conformal RT to be associated with radiation damage.[6] Similar dosimetric variables 
have been associated with treatment-related necrosis following repeat stereotactic radiosurgery for brain metastases.[23] Dose escalation in the recurrent setting should, therefore, be performed thoughtfully.

Several contemporary studies utilizing higher-dose reirradiation regimens have identified dose-response relationships in regard to treatment outcomes. The Italian Association of Radiation Oncology described 300 recurrent glioma patients retreated with a median biological effective dose (BED) of $43 \mathrm{~Gy}$. They identified reirradiation dose $>43$ Gy was associated with improved OS compared to < 43 Gy $(12.2$ months vs 6.7 months).[17] Veninga et al. identified improved OS for anaplastic astrocytoma patients receiving $\mathrm{BED}_{10}>55 \mathrm{~Gy}$ (10.8 months vs. 4.8 months).[24] Lee et al. described 36 recurrent glioma patients and found $>45$ Gy to be associated with improved OS. [25] Shen et al. reported 118 recurrent high-grade glioma patients and found improved OS was associated with > 41.4 Gy.[26] Lastly, Chapman et al. described 116 patients treated with a median $\mathrm{BED}_{10}$ of $47.25 \mathrm{~Gy}$ and reported that $\mathrm{BED}_{10}$ was associated with PFS but not OS.[27] Overall, these results lend support to the finding from our study that $\geq 50 \mathrm{~Gy}$ (CGE) was associated with improved outcomes on UVA and together suggest a possible benefit to reirradiation dose escalation of at least 43-50 Gy. It is important to note, however, that possible confounding clinical factors may have contributed to patients being selected to receive lower doses (e.g. performance status, tumor burden, or inability to dose escalate due to tumor-OAR proximity), and this may explain the exclusion of dose in our MVA model. Further prospective study of dose escalation is warranted.

Reports of PT-reirradiation for recurrent malignant glioma are scarce. Mizumoto et al. described 26 patients with recurrent malignant brain tumors, including 8 who received PT. The median reirradiation dose and OS of patients with glioblastoma $(n=5)$ were 30 CGE (range 30-60 CGE) and 18.3 months (range 3.9-48 months), respectively. No high-grade toxicities were reported. [20] Galle et al. reported 20 patients with recurrent malignant glioma treated with PT. Median dose and OS for patients with grade III and IV tumors were 59.4 CGE/24.9 months and 54 CGE/7.8 months, respectively. On UVA, reirradiation dose was associated with OS from diagnosis. Two patients developed radiation necrosis.[28] Lastly, Saeed et al. reported a multi-center experience using PT to treat recurrent glioblastoma, including 45 patients receiving a median dose of 46.2 CGE (range 25-60 CGE). The median PFS and OS were encouraging at 13.9 and 14.2 months, respectively. They also reported a modest incidence of late grade 3 toxicities at 8.3\%.[29] The overall incidence of grade 3 toxicity in our study was similar (5.6\%) and there were notably no grade 4-5 PT-related toxicities. These outcomes are particularly encouraging given the higher reirradiation doses used in this series. Based on these results, PT-reirradiation appears similarly effective but potentially favorable in terms of toxicity compared with photon reirradiation and may facilitate more aggressive salvage therapy.

Malignant gliomas are highly heterogeneous tumors with varying prognoses. Therefore, patient selection and use of molecular biomarkers are critical to identify patients who may benefit most from aggressive salvage therapies and advanced radiation modalities. In our study, survival curves for the $\geq 50$ Gy (CGE) cohort prominently separates around 9-12 months, suggesting patients with $>1$ year life expectancy would derive the greatest benefit from this treatment paradigm. In addition, future trials should also focus 
on QOL and neurocognitive preservation, domains that were not available for analysis in the present dataset. Prior studies have demonstrated more optimal QOL preservation for patients with recurrent glioblastoma treated with PT-reirradiation.[30] For patients with newly diagnosed low-grade glioma, PT is reported to preserve neurocognitive test scores, and even result in improved scores for left-sided tumors. $[31,32]$ Given the extended survival of many patients, particularly those with non-glioblastoma, the risk of neurocognitive deterioration should be considered when deciding on RT modality, and pencil-beam scanning PT may further preserve QOL and/or reduce toxicities compared with passive-scattering PT. Lastly, there remains no clear standard for dose and fractionation after recurrence; however, fractionated RT may be better suited for dose escalation to minimize the late effects on normal brain tissue given its inherent low $\alpha / \beta$ ratio.[33]

Despite the use of a prospectively-collected multi-institutional dataset and large sample size, several limitations to the current work are notable. There were limited molecular data, particularly for nonglioblastoma patients, which could more accurately stratify patient analysis. In addition, the database was unable to collect patterns of recurrence and detailed dosimetric parameters, including extent of field overlap and OAR doses. These specific factors are critical to optimal patient selection and more detailed outcomes analyses. More robust prospective evaluations of these factors will be needed on future prospective institutional trials. Lastly, PCG toxicity data relies on institutional evaluation and the database does not provide primary radiographic data. Therefore, rates of radiation necrosis and estimates of PFS has a degree of uncertainty given the difficulty in distinguishing pseudoprogerssion versus disease progression. Some patients did not continue regular follow-up at their proton center of treatment, potentially underestimating certain events.

In conclusion, our series reports 109 patients with recurrent malignant glioma who received PTreirradiation. This study demonstrates comparable efficacy and favorable tolerability relative to photon series. Dose escalation > 50 Gy may be more safety achievable with PT and further investigation is warranted for well-selected patients. Future studies are needed to quantify potential neurocognitive and QOL advantages of PT in the reirradiation setting and correlate cumulative OAR doses with risk of highgrade toxicities.

\section{Declarations}

Compliance with Ethical Standards

Funding: No funds, grants, or other support was received for this manuscript.

Conflicts of interest: CBS reports a Varian Medical Systems honorarium outside of the submitted work. MVM reports a Varian Medical Systems honorarium outside of the submitted work. The remaining authors have no relevant financial or non-financial interests to disclose.

Ethical approval: All procedures performed in studies involving human participants were in accordance with the ethical standards of the institutional and/or national research committee and with the 1964 
Helsinki declaration and its later amendments or comparable ethical standards.

Informed consent: The study was approved by each participating institutional review board. Informed consent was obtained for each patient enrolled on the PCG registry.

\section{References}

1. Ostrom QT, Cioffi G, Gittleman H et al (2019) CBTRUS Statistical Report: Primary Brain and Other Central Nervous System Tumors Diagnosed in the United States in 2012-2016. Neuro Oncol 21:v1v100. https://doi.org/10.1093/neuonc/noz150

2. McDonald MW, Shu H-KG, Curran WJJ, Crocker IR (2011) Pattern of failure after limited margin radiotherapy and temozolomide for glioblastoma. Int J Radiat Oncol Biol Phys 79:130-136. https://doi.org/10.1016/j.ijrobp.2009.10.048

3. Minniti G, Amelio D, Amichetti M et al (2010) Patterns of failure and comparison of different target volume delineations in patients with glioblastoma treated with conformal radiotherapy plus concomitant and adjuvant temozolomide. Radiother Oncol J Eur Soc Ther Radiol Oncol 97:377-381. https://doi.org/10.1016/j.radonc.2010.08.020

4. Seystahl K, Wick W, Weller M (2016) Therapeutic options in recurrent glioblastoma-An update. Crit Rev Oncol Hematol 99:389-408. https://doi.org/10.1016/j.critrevonc.2016.01.018

5. Flickinger JC, Deutsch M, Lunsford LD (1989) Repeat megavoltage irradiation of pituitary and suprasellar tumors. Int J Radiat Oncol Biol Phys 17:171-175. https://doi.org/10.1016/03603016(89)90385-4

6. Shepherd SF, Laing RW, Cosgrove VP et al (1997) Hypofractionated stereotactic radiotherapy in the management of recurrent glioma. Int J Radiat Oncol Biol Phys 37:393-398. https://doi.org/10.1016/s0360-3016(96)00455-5

7. Klein M, Heimans JJ, Aaronson NK et al (2002) Effect of radiotherapy and other treatment-related factors on mid-term to long-term cognitive sequelae in low-grade gliomas: a comparative study. Lancet 360:1361-1368. https://doi.org/10.1016/s0140-6736(02)11398-5

8. Douw L, Klein M, Fagel SS et al (2009) Cognitive and radiological effects of radiotherapy in patients with low-grade glioma: long-term follow-up. Lancet Neurol 8:810-818.

https://doi.org/10.1016/S1474-4422(09)70204-2

9. Correa DD, Shi W, Thaler HT et al (2008) Longitudinal cognitive follow-up in low grade gliomas. J Neurooncol 86:321-327. https://doi.org/10.1007/s11060-007-9474-4

10. Verma V, Rwigema J-CM, Malyapa RS et al (2017) Systematic assessment of clinical outcomes and toxicities of proton radiotherapy for reirradiation. Radiother Oncol J Eur Soc Ther Radiol Oncol 125:21-30. https://doi.org/10.1016/j.radonc.2017.08.005

11. Simone CB 2nd, Plastaras JP, Jabbour SK et al (2020) Proton Reirradiation: Expert Recommendations for Reducing Toxicities and Offering New Chances of Cure in Patients With 
Challenging Recurrence Malignancies. Semin Radiat Oncol 30:253-261.

https://doi.org/10.1016/j.semradonc.2020.02.007

12. Nieder C, Astner ST, Mehta MP et al (2008) Improvement, clinical course, and quality of life after palliative radiotherapy for recurrent glioblastoma. Am J Clin Oncol 31:300-305.

https://doi.org/10.1097/COC.0b013e31815e3fdc

13. Scoccianti S, Francolini G, Carta GA et al (2018) Re-irradiation as salvage treatment in recurrent glioblastoma: A comprehensive literature review to provide practical answers to frequently asked questions. Crit Rev Oncol Hematol 126:80-91. https://doi.org/10.1016/j.critrevonc.2018.03.024

14. Combs SE, Thilmann C, Edler $L$ et al (2005) Efficacy of fractionated stereotactic reirradiation in recurrent gliomas: long-term results in 172 patients treated in a single institution. J Clin Oncol Off J Am Soc Clin Oncol 23:8863-8869. https://doi.org/10.1200/JC0.2005.03.4157

15. Fogh SE, Andrews DW, Glass J et al (2010) Hypofractionated stereotactic radiation therapy: an effective therapy for recurrent high-grade gliomas. J Clin Oncol Off J Am Soc Clin Oncol 28:30483053. https://doi.org/10.1200/JC0.2009.25.6941

16. Adkison JB, Tomé W, Seo S et al (2011) Reirradiation of large-volume recurrent glioma with pulsed reduced-dose-rate radiotherapy. Int J Radiat Oncol Biol Phys 79:835-841.

https://doi.org/10.1016/j.ijrobp.2009.11.058

17. Navarria P, Minniti G, Clerici E et al (2019) Re-irradiation for recurrent glioma: outcome evaluation, toxicity and prognostic factors assessment. A multicenter study of the Radiation Oncology Italian Association (AIRO). J Neurooncol 142:59-67. https://doi.org/10.1007/s11060-018-03059-x

18. Kim EY, Yechieli R, Kim JK et al (2014) Patterns of failure after radiosurgery to two different target volumes of enhancing lesions with and without FLAIR abnormalities in recurrent glioblastoma multiforme. J Neurooncol 116:291-297. https://doi.org/10.1007/s11060-013-1290-4

19. Cuneo KC, Vredenburgh JJ, Sampson JH et al (2012) Safety and efficacy of stereotactic radiosurgery and adjuvant bevacizumab in patients with recurrent malignant gliomas. Int J Radiat Oncol Biol Phys 82:2018-2024. https://doi.org/10.1016/j.ijrobp.2010.12.074

20. Mizumoto M, Okumura T, Ishikawa E et al (2013) Reirradiation for recurrent malignant brain tumor with radiotherapy or proton beam therapy. Technical considerations based on experience at a single institution. Strahlenther Onkol 189:656-663. https://doi.org/10.1007/s00066-013-0390-6

21. Tsien C, Pugh S, Dicker adam et al (2019) ACTR-32. NRG ONCOLOGY RTOG 1205: RANDOMIZED PHASE II TRIAL OF CONCURRENT BEVACIZUMAB AND RE-IRRADIATION VS. BEVACIZUMAB ALONE AS TREATMENT FOR RECURRENT GLIOBLASTOMA. Neuro Oncol 21:vi20-vi20. https://doi.org/10.1093/neuonc/noz175.075

22. Bergman D, Modh A, Schultz L et al (2020) Randomized prospective trial of fractionated stereotactic radiosurgery with chemotherapy versus chemotherapy alone for bevacizumab-resistant high-grade glioma. J Neurooncol 148:353-361. https://doi.org/10.1007/s11060-020-03526-4

23. McKay WH, McTyre ER, Okoukoni C et al (2017) Repeat stereotactic radiosurgery as salvage therapy for locally recurrent brain metastases previously treated with radiosurgery. J Neurosurg 127:148- 
156. https://doi.org/10.3171/2016.5.JNS153051

24. Veninga T, Langendijk HA, Slotman BJ et al (2001) Reirradiation of primary brain tumours: survival, clinical response and prognostic factors. Radiother Oncol J Eur Soc Ther Radiol Oncol 59:127-137. https://doi.org/10.1016/s0167-8140(01)00299-7

25. Lee J, Cho J, Chang JH, Suh CO (2016) Re-Irradiation for Recurrent Gliomas: Treatment Outcomes and Prognostic Factors. Yonsei Med J 57:824-830. https://doi.org/10.3349/ymj.2016.57.4.824

26. Shen CJ, Kummerlowe MN, Redmond KJ et al (2018) Re-irradiation for malignant glioma: Toward patient selection and defining treatment parameters for salvage. Adv Radiat Oncol 3:582-590. https://doi.org/10.1016/j.adro.2018.06.005

27. Chapman $\mathrm{CH}$, Hara JH, Molinaro AM et al (2019) Reirradiation of recurrent high-grade glioma and development of prognostic scores for progression and survival. Neuro-oncology Pract 6:364-374. https://doi.org/10.1093/nop/npz017

28. Galle JO, McDonald MW, Simoneaux V, Buchsbaum JC (2015) Reirradiation with Proton Therapy for Recurrent Gliomas. Int J Part Ther 2:11-18. https://doi.org/10.14338/THEIJPT-14-00029.1

29. Saeed AM, Khairnar R, Sharma AM et al (2020) Clinical Outcomes in Patients with Recurrent Glioblastoma Treated with Proton Beam Therapy Reirradiation: Analysis of the Multi-Institutional Proton Collaborative Group Registry. Adv Radiat Oncol 5:978-983. https://doi.org/10.1016/j.adro.2020.03.022

30. Scartoni D, Amelio D, Palumbo P et al (2020) Proton therapy re-irradiation preserves health-related quality of life in large recurrent glioblastoma. J Cancer Res Clin Oncol 146:1615-1622. https://doi.org/10.1007/s00432-020-03187-w

31. Sherman JC, Colvin MK, Mancuso SM et al (2016) Neurocognitive effects of proton radiation therapy in adults with low-grade glioma. J Neurooncol 126:157-164. https://doi.org/10.1007/s11060-0151952-5

32. Verma V, Simone CB 2nd, Mishra MV (2018) Quality of Life and Patient-Reported Outcomes Following Proton Radiation Therapy: A Systematic Review. J Natl Cancer Inst 110:. https://doi.org/10.1093/jnci/djx208

33. Shanker M, Chua B, Bettington C et al (2019) Re-irradiation for recurrent high-grade gliomas: a systematic review and analysis of treatment technique with respect to survival and risk of radionecrosis. Neuro-oncology Pract 6:144-155. https://doi.org/10.1093/nop/npy019

\section{Figures}



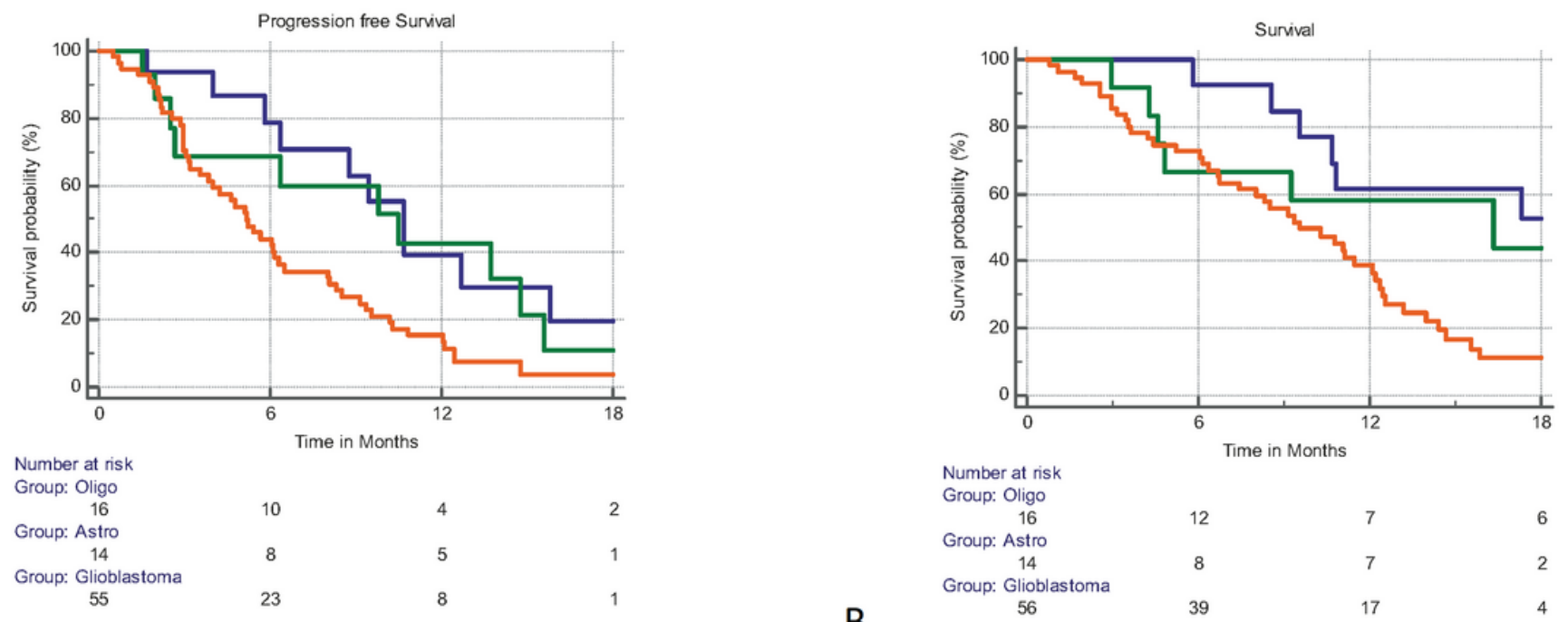

A

B

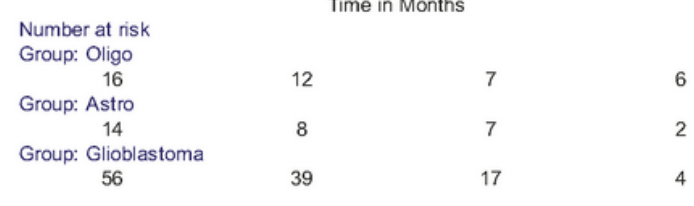

Figure 1

Kaplan Meier curves demonstrating (a) Progression-Free Survival and (b) Overall Survival by histology for patients with 1 prior course of radiotherapy $(n=102)$ (orange $=$ glioblastoma, green $=$ astrocytoma, blue $=$ oligodendroglioma).

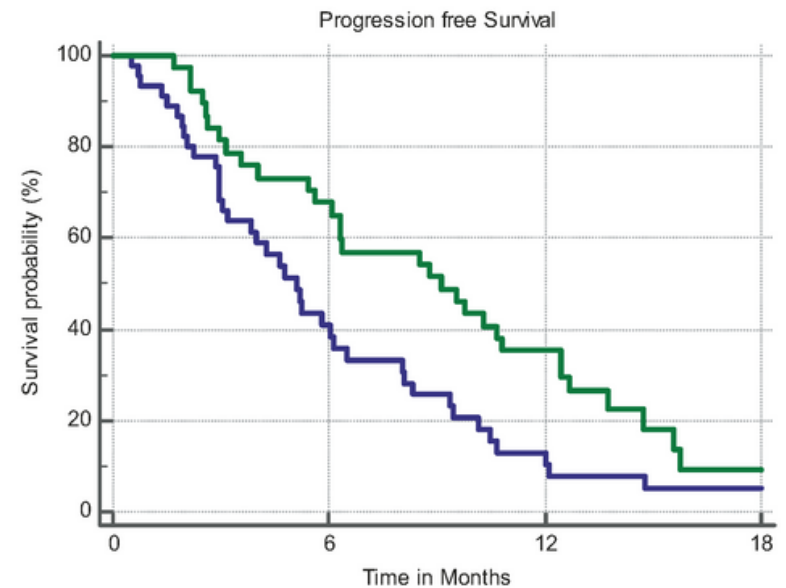

Number at risk

Group: $\geq 50$ Gy

Group: $<50$ Gy

39

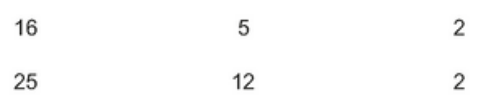

A

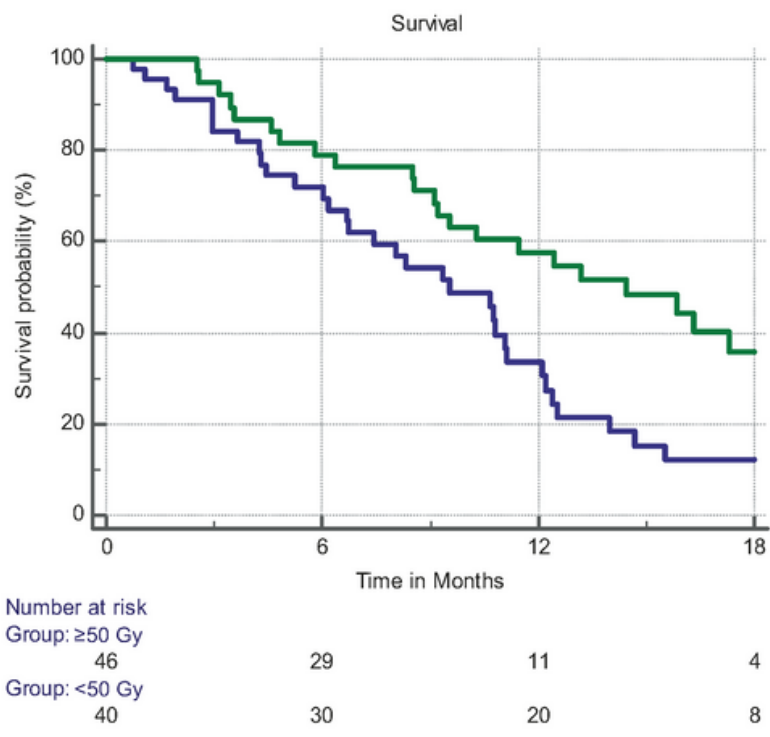

B

Figure 2 
Kaplan Meier curves demonstrating (a) Progression-Free Survival and (b) Overall Survival by reirradiation dose $>50$ Gy vs $<50$ Gy. (Green $=>50$ Gy, Blue $=<50$ Gy) $($ CGE). 